\title{
$\begin{array}{lllllllllllllll}\text { F } & \text { E } & \text { A } & \text { T } & \text { U } & \text { R } & \text { E } & & \text { A } & \text { R } & \text { T } & \text { I } & \text { C } & \text { I } & \text { E }\end{array}$
}

\section{Tracking and Surface Discharge at the Oil-Pressboard Interface}

\author{
Key words: transformer, partial discharge, electric double layer, tracking, static electrification
}

\section{Introduction}

Great care is taken when designing and manufacturing large oil-filled transformers. One design requirement is to ensure that all conducting surfaces have blended geometries and are covered by solid insulation material, typically a cellulose material in the form of paper or pressboard. This prevents the emission of electronic charge that will occur from any bare conducting surfaces according to the modified Schottky process when a conductor is under high electrical stress [1]. The solid insulation effectively adds an extra barrier to charge emission. However, other phenomena can introduce charge into the insulation system under certain conditions. For example, space charge can form at an oil-pressboard interface under a high electric field due to interfacial polarization arising from the difference in permittivity between adjacent materials [2]. Partial discharges (PD) are another source of electrical charge generation. Partial discharges may occur in solid, liquid, and gaseous insulations wherever there are sharp changes or discontinuities within the insulation medium and where the electric field is sufficiently high to exceed the local breakdown value. Surface contamination is a likely site for PD due to the presence of inclusions and changes in bulk material. Finally, oil flowing across pressboard can build up static charge on the pressboard surface, a phenomenon referred to as static electrification but also known as flow or streaming electrification [3].

The movement of excessive charge over solid insulation may result in mechanical and chemical damage to material through thermal and electronic action. For solid insulators, 2 different forms of damage are generally classified. Surface damage is termed tracking, whereas damage through the bulk of the (solid) material is termed treeing. In both cases, the damage results in a permanent electrically conductive path that reduces the effective voltage withstand of the material. Tracking has traditionally been associated with solid-air interfaces and polymeric insulators [4]. However, tracking is now recognized as a significant feature at oil-cellulose interfaces and is considered a possible failure mode in large oil-filled transformers [5]. The term creeping discharge has been coined to describe the characteristic irreversible tree-like patterns occurring on the interface as a result of these surface discharges [6]. Surface tracking can deteriorate

\author{
P. M. Mitchinson, P. L. Lewin, \\ and B. D. Strawbridge \\ School of Electronics and Computer Science, Uni- \\ versity of Southampton, Southampton SO17 1BJ, \\ $U K$
}

\section{P. Jarman}

National Grid, National Grid House, Warwick Technology Park, Gallows Hill, Warwick CV34 6DA, UK

the surface resistance to such an extent that a sudden electrical discharge or breakdown can occur if the electrical stress is high enough. The sudden electrical breakdown is referred to as surface flashover, thus implying that the breakdown occurs in the medium above the surface [7]. Substrate material, surface finish, surface contamination, condition of fluid material, temperature, pressure of fluid, level of applied electric stress, and duration of application have been identified as the significant factors leading to surface tracking and/or flashover [8]. The oil-pressboard interface is also acknowledged as one of the main locations for interfacial polarization, as well as static electrification. Clearly, the oil-pressboard interface plays a significant role in the life of a transformer. However, many issues remain unanswered as to 
the exact nature of tracking. How can tracking in a liquid environment be reliably produced and quantified? What role does static electrification play in tracking, if at all? What defines the oil-pressboard interface?

This paper addresses some of these questions. First, the various experimental methods of producing surface tracking are reviewed, and a new approach proposed. Static electrification is also considered against a new interpretation of the oil-pressboard interface. Finally, the results of some tracking experiments on standard pressboard type Transformerboard TIV [9] are examined when using the new approach to produce surface tracking.

\section{The Measurement and Production of Surface Tracking}

The measure of the resistance of solid insulation materials to tracking in air is determined by the Comparative Tracking Index standard [10]. Two tests are available, and these use either a horizontal surface or an inclined slope. The tests employ a liquid contaminant, sprayed in droplet form onto the solid surface, to promote tracking. The standard considers only air as the fluid medium above the surface, and this raises the issue as to how to quantify surface tracking at the interface between a liquid and a solid.

For liquid-solid interfaces, several different methods have historically been used to create surface discharge. One common approach is to implement a point-plane arrangement [11], [12]. A needle is connected to a high voltage source and placed on or near the surface of the pressboard. This results in an intense space charge around the tip and causes electrical discharges to occur around the needle tip, leaving radial tracking patterns on the surface of the solid insulation. However, the needle produces a directional and intense electrical field, which can lead to a rapid electrical failure through the bulk medium when using pressboard as the dielectric. Another approach is to use a parallel plane-plane method with pressboard placed perpendicularly between 2 electrodes and with a small point source created on the surface of one of the plane electrodes and adjacent to the pressboard [13]. This method overlooks the fact that the electric field associated with the electrode arrangement is not separated from the enhanced electrical field arising from the discharge source. This makes the effects due to the localized discharge source difficult to separate from the effects of the resultant electrical field associated primarily with the electrodes.

A different method has been developed in the Tony Davies High Voltage Laboratory to overcome the difficulties of the traditional approaches [14]. This is an adaptation to the Comparative Tracking Index configuration; a needle discharge source is placed at an acute angle to the pressboard at some distance from an earthed conductor also placed on the pressboard, with the system immersed in oil. The acute angle of the needle ensures that charge arising at the needle tip is directed along the surface rather than through the bulk of the pressboard. This method has been found to reliably produce surface discharge when the voltage source ranges between PD inception and surface flashover. Three conclusions were drawn from this approach. The first is that the degree of damage due to PD is clearly time and voltagelevel dependent. This means that surface tracking can be established and sustained on the oil-pressboard interface for a considerable time without electrical breakdown.

The second conclusion is that PD inception/extinction is insensitive to the gap distance from earth, suggesting that surface discharge is a localized phenomenon. Surface flashover and intrinsic oil voltage breakdown are sensitive to the gap distance, indicating that these mechanisms are associated with bulk material properties.

The third conclusion is that the presence of the solid surface depresses the intrinsic voltage withstand of the bulk oil medium so that surface flashover occurs at a lower value than the bulk medium properties would predict. The differences in the features observed between tracking and surface flashover suggest that these events occur at 2 different layers of the surface and that the structure plays a role in voltage depression from the intrinsic voltage withstand of the bulk oil.

\section{Static Electrification}

The transformer industry first became aware of the problem of static electrification in the 1970s following examination of transformer failures [3]. Static electrification is now accepted to be due to the electric double layer (EDL) at the oil-pressboard interface [15], [16]. The EDL, first proposed by Helmholtz, is the variation in potential across a surface interface between 2 different materials. It is analogous to the depletion zone in the transition region at the junction between $\mathrm{p}-\mathrm{n}$ semiconductors. In the case of the solid-liquid interface, the EDL model describes a homogenous solid surface with the EDL existing in the liquid layer at a thickness of no more than 1 or 2 molecules next to it [17]. The model has been expanded to the Guoy-Chapman Stern model, which describes 2 layers: the thin Stern layer, which carries the majority of the charge, and a diffuse layer where charged liquid molecules are less densely spaced and more mobile [18].

The mechanism for static electrification is the interaction of the oil with the pressboard as the oil moves across the surface of the pressboard. The oil develops a charge depending on the surface over which it travels. If the oil travels over a region where there is an availability of free electrons (i.e., a conducting surface), then the oil acquires negative charge. Alternatively, if the oil travels across pressboard, the cellulose hydroxyl groups tend to acquire a negative charge from the moving oil, leaving the oil positively charged [17]. The oil relaxes the charge as it approaches earthed surfaces. If the relaxation does not eliminate charge quickly enough, a static electrical field can be established, which may end in a static electrical discharge [19], [20]. This phenomenon has been reported as a significant factor in some transformer failures [3], [16], [21]. Static electrification is a function of many parameters including temperature and moisture but primarily the pressboard surface structure and oil condition, specifically the oil electrical charging tendency [22]. Dry oil, dry pressboard, and surface roughness increase the likelihood of static electrification [3], [15], and it has been suggested that the presence of carboxylic groups also increases static electrification [20]. This has led to the use of oil additives to counteract it [23], [24]. Current research on static electrification focuses 
on the chemistry of pressboard rather than mechanical surface features such as porosity and surface roughness, which are only mentioned in passing [25].

It is clear that interfacial polarization and static electrification have the same roots through the charging tendency of different materials when in close proximity. This raises the issue of the possibility of an interaction between the electric field, which causes interfacial polarization, and the static electric field created in the EDL produced by oil flow over an insulation surface. The link is the solid-liquid interface. A closer inspection of this interface between oil and pressboard suggests that it is more complicated than first assumed.

\section{Pressboard and the Oil-Pressboard Interface}

The use of the word solid to describe pressboard conveys the sense of a homogenous and dense material. Pressboard is in fact a light, fibrous, and porous material [26]. In use, pressboard is first dried and then impregnated with mineral oil under high vacuum conditions. This process removes moisture and gasses from the fibrous structure and refills the interstices with oil molecules. This proven method has been the key in extending the life of pressboard as an insulating material. The measure of impregnation is defined by oil absorption by mass, which is typically $13 \%$ for TIV Transformerboard [9], although it varies according to material and surface finish [27]. After the impregnation process, the oil pressboard insulation medium effectively forms a composite insulation structure with the oil.

A casual look at the oil-pressboard interface suggests that the interface changes abruptly from bulk pressboard to the bulk oil. However, a closer examination of the surface of pressboard reveals that the situation is rather more complex. Pressboard is manufactured in a range of surface finishes from a smooth (i.e., calendered) finish to a textured (i.e., cloth) finish [28]. Figure 1 shows the surface textured finish of TIV Transformerboard, which is characterized by an array of small and large dimples orthogonal to each other and set in the bulk fibrous backdrop. A microtome section reveals the edge to be quite irregular with protruding fibers and characterized by a transition region of approximately $350 \mu \mathrm{m}$ before the bulk volume of pressboard (Figure 2). (Note: Figures 1 and 2 have been captured using a green filter to enhance features.)

The oil-pressboard interface is, therefore, not the clean edge it appears to be from a distance; in reality, there is an ill-defined interface comprising a transition region between bulk oil/pressboard composite and bulk oil. In this transition region, the ratio of oil must change from the typical value of $13 \%$ (i.e., the bulk value of oil adsorption) toward $100 \%$ as the material structure changes from the bulk oil/pressboard composite medium toward the bulk oil medium. This oil-pressboard transition zone is further complicated by the presence of the dimples and individual fibers, as shown in Figure 2. The liquid boundary layer, as defined by conventional boundary layer theory, resides just outside the "solid" surface. However, the ill-defined pressboard edge and the presence of raised fibers suggest that the extent of both

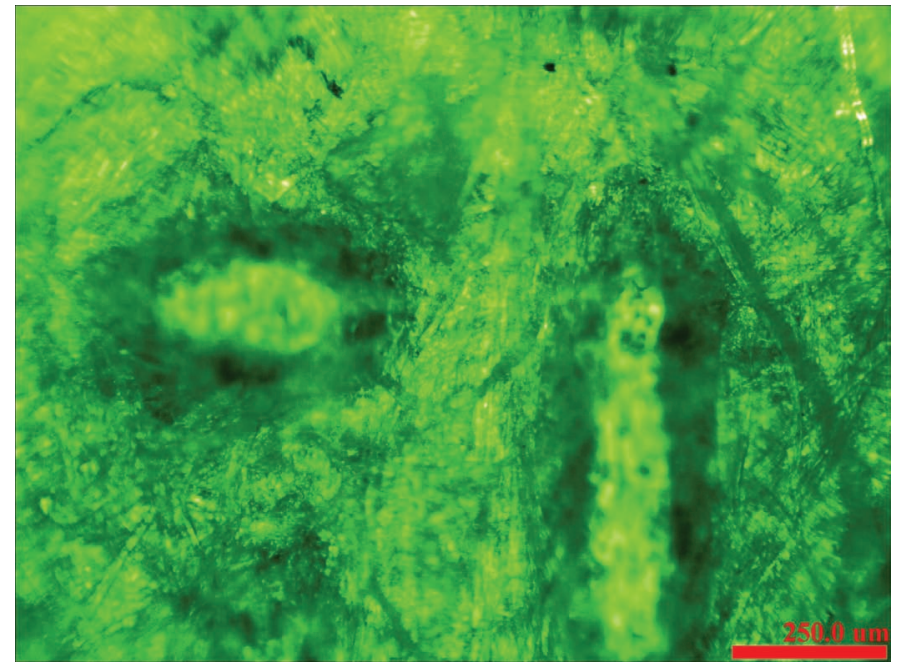

Figure 1. Morphology of dry pressboard surface at 200× magnification.

the EDL and the liquid boundary layer could be greater than theory might indicate.

The classical mechanical model, with nonpolar "solid" pressboard and liquid mineral oil molecules, defines a liquid boundary layer formed by weak Van de Waals forces between the covalent molecules. The liquid molecules, closest to the "solid" medium, stick to the solid and create the so-called no-slip layer, which is equivalent to the Stern layer in the Guoy-Chapman Stern model (thus linking the Stern layer in the EDL model to the no-slip layer in the mechanical model). These molecules are fixed, and there is a gradual transition from stationary oil molecules to the oil molecules in the free stream, with the region in between forming the boundary layer (thus linking the diffuse layer in the EDL model to the boundary layer in the mechanical model). The boundary layer is small in thickness where laminar flow may occur and increases in thickness for turbulent flow. A

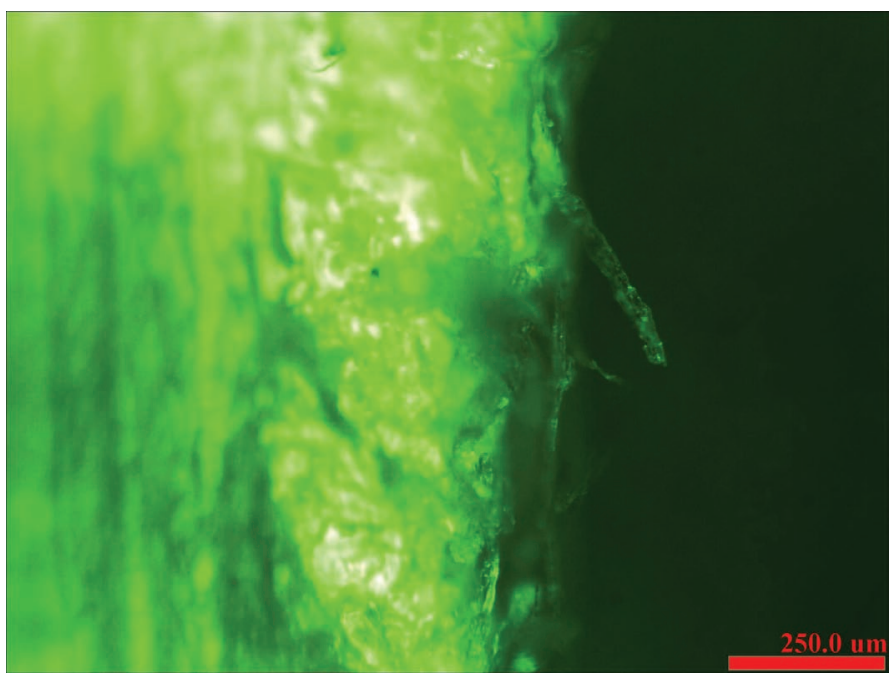

Figure 2. Microtome of pressboard edge at $200 \times$ magnification 


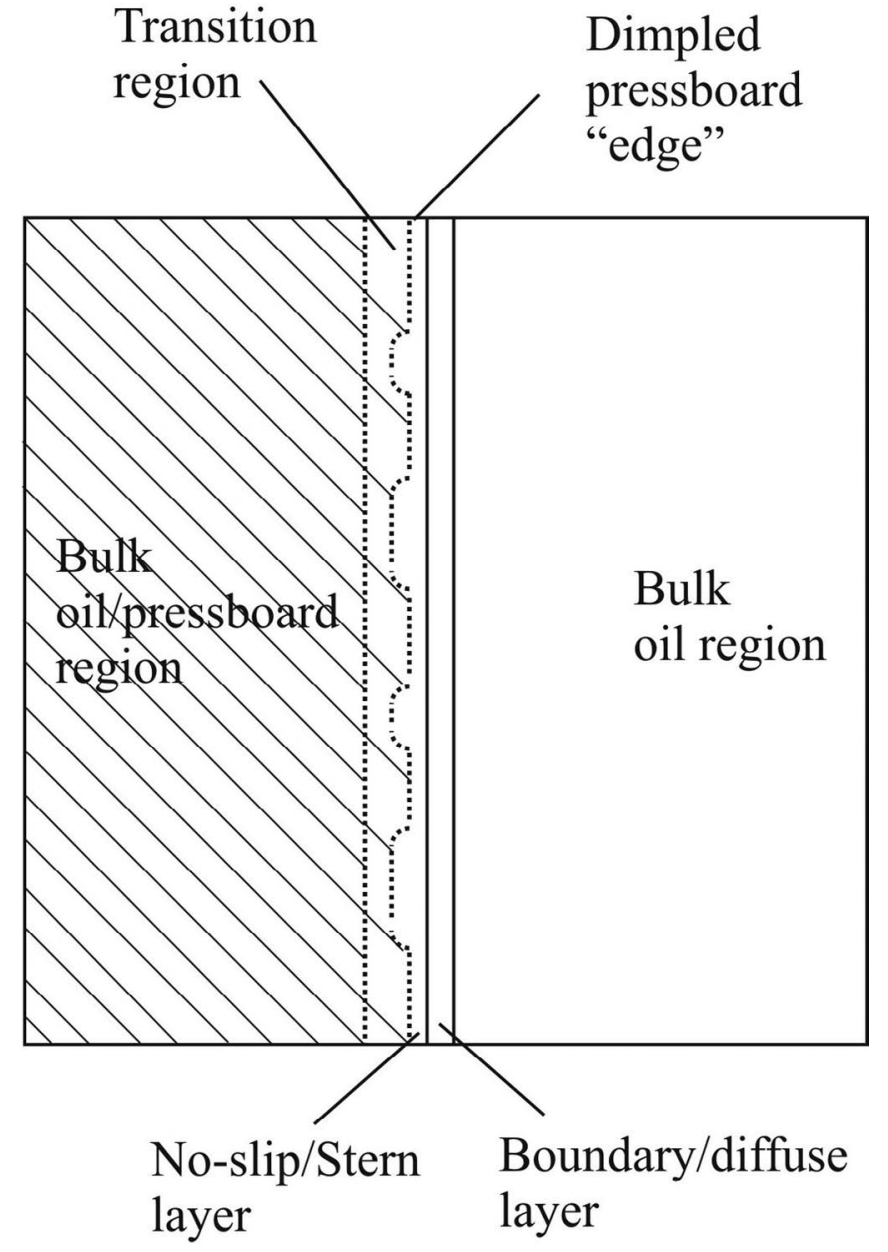

Figure 3. A new model of the oil-pressboard interface. rough surface increases turbulent flow and the thickness of the boundary layer, whereas a smooth surface diminishes the turbulence and, hence, the thickness of the boundary layer. A new model of the oil-pressboard interface could be described as the liquid boundary (diffuse) layer (incorporating the no-slip /Stern layer), next to the transition region, which merges into the bulk oil-pressboard composite structure (Figure 3).

\section{Features of Surface Tracking Observed Using the New Approach}

A series of experiments using the new needle bar approach were undertaken on oil-impregnated pressboard conditioned to 3 to $4 \%$ moisture content, with the needle discharge source positioned $35 \mathrm{~mm}$ from the earth bar and with the voltage raised to between $30 \mathrm{kV}$ and $40 \mathrm{kVrms}$. Partial discharge inception was reliably produced at this level, leading to sustained surface discharge with surface flashover/breakdown initiated when the voltage was raised above $40 \mathrm{kV}$. The experiments were qualitative rather than quantitative in nature; the objective was to examine surface tracking on the oil-pressboard interface. Data collection was achieved using a standard 35-mm digital camera. Partial discharge activity was monitored using the Omicron MPD600 PD system. A typical phase resolved plot of PD activity indicating surface tracking activity taken over $30 \mathrm{~s}$ is given in Figure 4. Some of the significant tracking and breakdown events, captured by the camera, are shown in Figures 5 to 8 .

\section{Discussion}

The Figures illustrate different features in the surface tracking/breakdown process. First, moist transformer board has to be used to initiate PD. It was found to be extremely difficult to produce PD with dried pressboard (i.e., $0.5 \%$ moisture), thus indicating that the presence of the moisture is a significant factor in the tracking process. Once PD is initiated, the first promi-
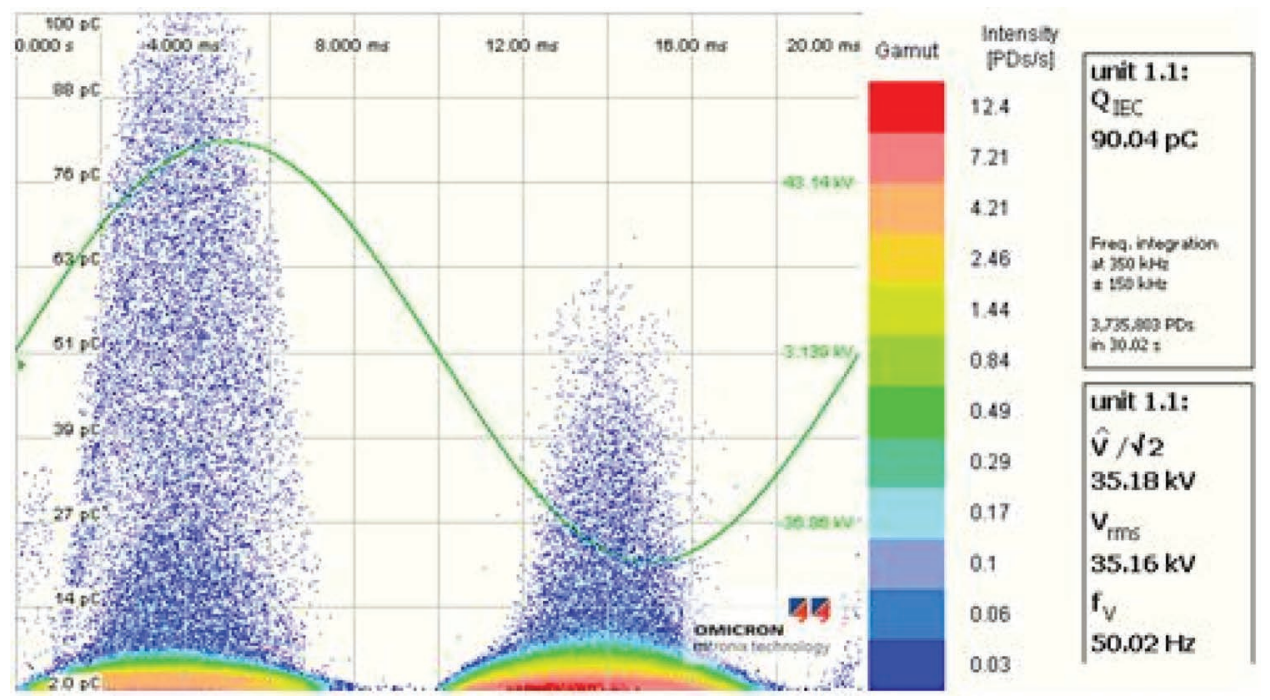

Figure 4. Phase resolved plot with needle bar at $35 \mathrm{kV}$ and 35-mm gap. 


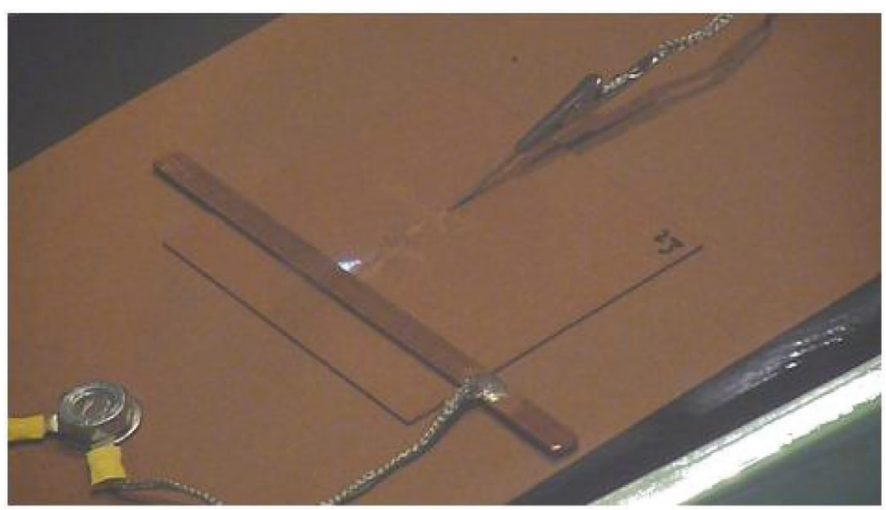

Figure 5. Initial growth of white marks and discharges in oil at the earth bar.

nent feature to be observed is the growth of white marks on the pressboard surface. The white marks grow initially from the discharge source toward the earth bar (Figure 5). These marks are indicative of a drying process as liquid in the pressboard is expelled with the injection of charge, resulting in localized reactions as evidenced by the evolution of smoke and gas. The marks develop into the characteristic fan-shaped pattern within 6 to 12 hours depending on voltage level (Figure 6). Black marks, indicating carbonization of oil/pressboard in the pressboard, follow the tracks of the white marks but do not necessarily extend the whole distance. Another feature is the random appearance of smoky particulates and gas bubbles that arise from the discharge tip. Gas bubbles may also appear anywhere along the white tracking marks as seen in Figure 6. Another feature occurs when the white marks nearly reach the earth bar. Small bluish flashes emanate from the earth bar to connect to the white marks. These flashes are clearly located in the oil above the surface of the pressboard (Figure 5). Another phenomenon is the full-length discharge that temporarily bridges the full distance from discharge tip to the earth bar without causing full electrical breakdown (Figure 7). In this case, 2 colors are clearly visible: an orange glow that occurs at the pressboard surface over the majority of the discharge length and bluish discharges at the earth bar. The most significant event is when the potential at the

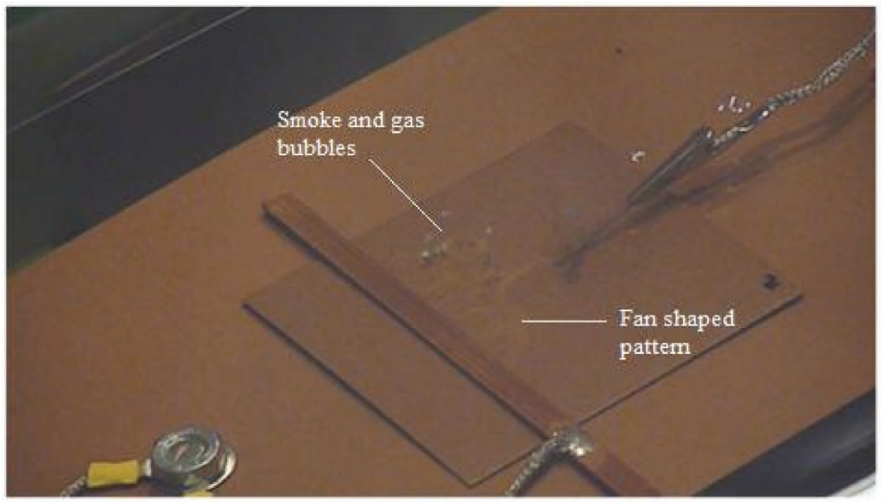

Figure 6. Growth of fan pattern from the discharge source and remote gassing.

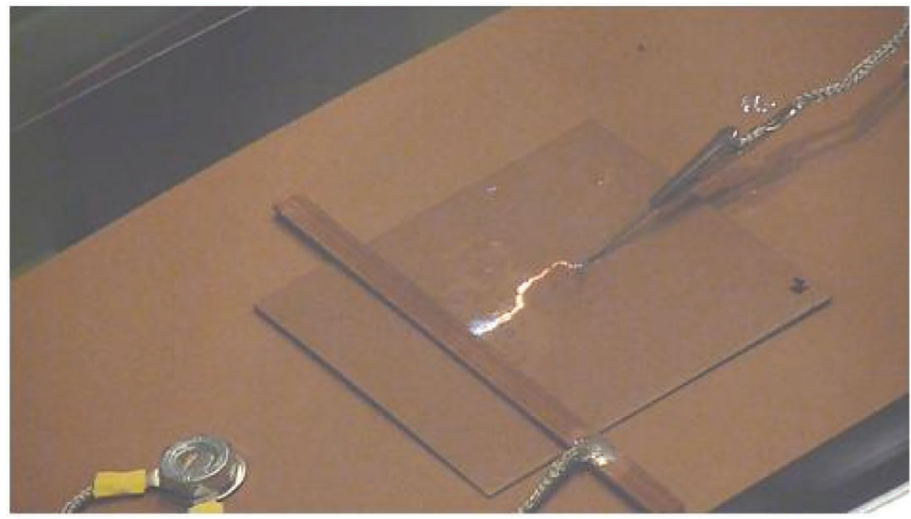

Figure 7. Full-length surface discharge without breakdown.

discharge source is increased to the system breakdown level. An instantaneous flashover occurs that is quite different from the full-length surface discharge. The breakdown discharge is more intensive and results in the evolution of significant amounts of smoky particulates and gas (Figure 8). However, the pressboard often remains largely undamaged, indicating that the discharge occurs in the oil layer. This shows that the term surface flashover is indeed an accurate description.

The occurrence of 2 distinct types of discharge, i.e., a slow surface tracking type with significant surface changes and a quick flashover type in the oil layer above the pressboard, suggests that 2 paths are available for electronic transport. The first path is along the transition zone/EDL/no-slip region at the oilpressboard interface where the mingling of oil, pressboard, and other species, such as water, provides a region for space charge to accumulate and drift toward the region of lower field intensity. The second path is in the free-oil boundary layer where the increased mobility of the oil allows the propagation of a breakdown discharge at higher energy levels.

This might explain why flashover results at a lower voltage than would occur with the same oil gap separation but without pressboard as reported in the literature [14], [29]. The volume created by the transition zone/EDL at the oil pressboard, with the consequent higher permittivity than bulk oil, permits more

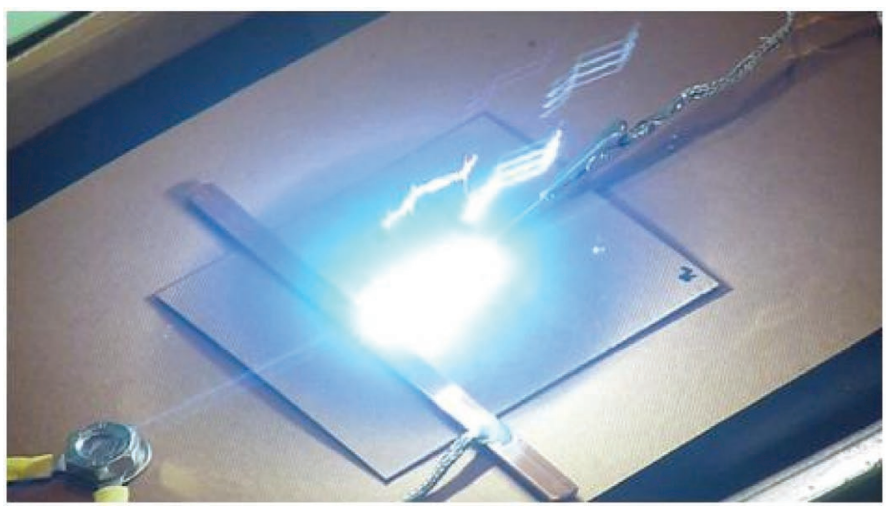

Figure 8. Flashover at $45 \mathrm{kV}$. 
charge to accumulate for a given voltage than for the bulk oil medium. Space charge is thus able to progress deeper along the pressboard surface, which results in breakdown at a lower value than would be predicted with the bulk oil volume alone.

\section{Conclusions}

The oil-pressboard interface is more complex than a cursory look would suggest. The interface is better modeled as a layered structure that takes into account the EDL/oil boundary layer, and the graduated nature of the pressboard transition region into the bulk pressboard oil composite. Moisture clearly plays an important role at the interface.

The complex nature of the interface with a layered structure of oil and pressboard suggests that 2 separate paths are available for charge transport depending on the energy level of the discharge source.

One path is the EDL and oil/pressboard transition zone and is associated with lower energy surface discharges and tracking. This path allows space charge to drift under the influence of the electric field causing the degradation known as tracking. The second path is the oil boundary layer which is associated with higher energy breakdown known as surface flashover.

The observations confirm that, once tracking paths are established, significant electrical discharges can occur without breakdown. These produce gassing and particulate degradation by-products, which are common measurements used by the conditioning monitoring industry.

Interfacial polarization is associated with the mismatch of 2 media, and static electrification is associated with the liquid boundary layer and oil flowing across a solid surface. This suggests that there might be a link between interfacial polarization and static electrification. It raises the intriguing issue of the possibility of an interaction between the 2 when a system is under both high electric stress and high fluid flow rates. Further work is also required to study both the effects of fluid flow and the influence of smoothness afforded by calendered surfaces on both tracking and flashover under these conditions.

\section{Acknowledgments}

The authors gratefully acknowledge the support of National Grid, UK, for project funding and Weidmann Electrical Technology, Switzerland, and Whiteley Limited, UK, for the supply of Transformerboard.

\section{References}

[1] E. O. Forster and G. J. Fitzpatrick, "Electrical breakdown in dielectric liquids," Physics Technol., vol. 16, no. 6, Nov. 1985.

[2] R. Liu, C. Tornkvist, and K. Johansson, "Space charge distribution in composite oil/cellulose insulation," in Conference on Electrical Insulation and Dielectric Phenomena, Arlington, TX, pp. 316-321, Oct. 1994.

[3] D. W. Crofts, "The static electrification phenomena in power transformers," IEEE Trans. Electr. Insul., vol. 23, no. 1, Jan. 1988.

[4] N. Yoshimura, S. Kumagai, and B. Du, "Research in Japan on the tracking phenomenon of electrical insulating materials," IEEE Electr. Insul. Mag., vol. 13, no. 5, Sept./Oct. 1997.

[5] V. V. Sokolov, "Understanding failure modes of transformers," in Proceedings of the Euro TechCon, Chester, UK, pp. 43-65, 2005.

[6] V. Sokolov, Z. Berler, and V. Rashkes, "Effective methods of assessment of insulation conditions in power transformers: A view based on practical experience," in Proceedings: Electrical Insulation Conference, Cincinnati, OH, pp. 659-667, Oct. 1999.

[7] L. L. Alston, High voltage technology, London, UK: Oxford University Press, 1968, pp. 4-5.

[8] N. Yoshimura, S. Kumagai, and B. Du, "Research in Japan on the tracking phenomenon of electrical insulating materials," IEEE Electr. Insul. Mag., vol. 13, no. 5, Sept./Oct. 1997.

[9] Transformerboard TIV Product Data Sheet, Weidmann Systems International.

[10] Method for the Determination of the Proof and the Comparative Tracking Indices of Solid Insulating Material, BS EN 60112: 2003.

[11] G. Berg and L. Lundgaard, "Discharges in combined oil/paper insulation," in Proceeding of the 1999 IEEE 13th International Conference on Dielectric Liquids, Nara, Japan, pp. 144-147, July 1999.

[12] C. Dervos, P. D. Bourkas, E. A. Kayafas, and I. A. Stathopulos, "Enhanced partial discharges due to temperature increase in the combined system of a solid-liquid dielectric," IEEE Trans. Electr. Insul. vol. 25, no. 3 June, 1990.

[13] M. Krins, H. Borsi, and E. Gockenbach, "Impact of carbon particles on the impulse flashover behavior of different solid/liquid interfaces in a non-uniform field," in Proceedings of 1998 International Symposium on Electrical Insulating Materials, Toyohashi, Japan, pp. 363-368, Sept. 1998.

[14] P. M. Mitchinson, P. L. Lewin, G. Chen, and P. Jarman, "A new approach to the study of surface discharge on the oil-pressboard interface," in IEEE International Conference on Dielectric Liquids, 2008, Poitiers, France, pp. 186-189, 2008.

[15] J. Zhang and L. J. Cao, "The study on flow electrification of oil-cellulose insulating system in large power transformer," in Proceedings of the International Conference on Energy Management and Power Delivery, Singapore, pp. 416-427, Nov. 1995.

[16] O. Moreau, G. Artana, H. Romat, and G. Touchard, "A contribution to modelling and experimental studies of flow electrification in transformers," 12th International Conference on Conduction and Breakdown in Dielectric Liquids, Rome, Italy, pp. 405-410, 1996.

[17] I. A. Metwally, "Influence of the solid insulating phase on streaming electrification of transformer oil," IEEE Trans. Dielectr. Electr. Insul., vol. 4, no. 3, June 1997.

[18] M. El-Adawy, J. M. Cabaleiro, T. Paillat, O. Moreau, and G. Touchard, "Experimental determination of space charge density associated with flow electrification phenomenon: Application to power transformers," $J$. Electrostatics, vol. 67, no. 2-3, pp. 354-358, May 2009.

[19] P. Mas, T. Paillat, O. Moreau, and G. Touchard, "Flow electrification in power transformers: Temperature influence on space charge distribution and charge accumulation in pressboard," J. Electrostatics, vol. 51-52, May 2001.

[20] T. Paillat, N. Charvet, O. Moreau, G. Mortha, Y. Bertrandy, and G. Touchard, "Influence of pressboard ionizable groups on static electrification in power transformers," in Conference on Electrical Insulation and Dielectric Phenomena, Cancun, Mexico, pp. 180-183, Oct. 2002.

[21] A. Sierota and J. Rungis, "Electrostatic charging in transformer oils," IEEE Trans. Dielectr. Electr. Insul., vol. 1, no. 5, Oct. 1994.

[22] L. Peyraque, A. Béroual, and F. Buret, "Static electrification of pressboard/oil interface and transient phenomena," IEEE Trans. Dielectr. Electr. Insul., vol. 5, no. 3, June 1998.

[23] E. Moreau, T. Paillat, and G. Touchard, "Flow electrification in high power transformers: BTA effect on pressboard degraded by electrical discharges," IEEE Trans. Dielectr. Electr. Insul., vol. 10, no. 1, Feb. 2003.

[24] A. Bourgeois, G. Mortha, T. Paillat, O. Moreau, and Y. Bertrand, "Flow electrification in power transformers: Study of a potential remedy," IEEE Trans. Dielectr. Electr. Insul., vol. 13, no. 3, June 2006.

[25] T. Paillat, L. Onic, O. Moreau, Y. Bertrand, G. Mortha, N. Charvet, and G. Touchard, "Influence of pressboard physio-chemical composition on static electrification in power transformers," IEEE Trans. Ind. Appl., vol. 39, no. 2 Mar./Apr. 2003.

[26] K. Giese, "The effect of cellulose insulation quality on electrical intrinsic strength," IEEE Electr. Insul. Mag., vol. 10, no. 5, Sept./Oct. 1994.

[27] P. K. Poovamma and R. Jagadish, "Influence of surface roughness and thickness of pressboard on the charging characteristics of transformer oil," in International Symposium on Electrical Insulation, pp. 526-529, June 1994. 
[28] Specification for Pressboard and Presspaper for Electrical Purposes. Specifications for Individual Materials. Requirements for Pressboard, Types B.0.1, B.2.1, B.2.3, B.3.1, B.3.3, B.4.1, B.4.3, B.5.1, B.6.1 and B.7.1, BS EN 60641-3-1:1994, IEC 60641-3-1:1992.

[29] L. Lundgaard, K. Herstad, M. U. Anker, and J. Sletbak, "Flashover along solid surfaces parallel to the electric field in liquid insulation at $50 \mathrm{~Hz}$," CIGRE Proceedings, Paris, France, 1986.

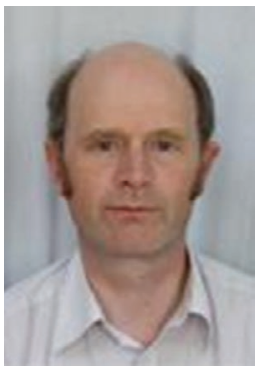

Mark Mitchinson trained as a marine engineer and then studied electrical and electronic engineering, gaining a BSc (Hons) at Loughborough University of Technology in 1985 . He has 20 years of engineering experience in industry including communications digital design, component design, and technical management in the volume manufacturing industry for small power and high frequency signal transformers. $\mathrm{He}$ is a chartered engineer and a member of both the IET and the IEEE. He was awarded a Doctorate in Philosophy by the University of Southampton in 2008 for a study of surface tracking in the inter-phase region of large liquid-filled transformers. He is currently a research fellow at the University of Southampton. His research interests include electric field modeling, discharge mechanisms, measurement techniques, materials, design for manufacturability, HV equipment and insulation condition monitoring, lifetime prediction, and failure analysis.

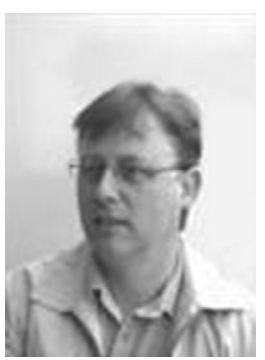

Paul L. Lewin (M '05, SM '08) was born in Ilford, Essex, in 1964. He received the BSc (Hons) and PhD degrees in electrical engineering from the University of Southampton, UK, in 1986 and 1994, respectively. He joined the academic staff of the university in 1989 and at present is the Reader in Electrical Power Engineering in the School of Electronics and Computer Science. He is also manager of the Tony Davies High Voltage Laboratory. His research interests include condition monitoring of $\mathrm{HV}$ cables and plant, surface charge measurement, HV insulation/dielectric materials, and applied signal processing. Since 1996, he has received funding and grants in excess of $£ 7.5 \mathrm{M}$, supervised 20 graduate students to successful completion of their doctoral theses, and published over 220 refereed conference and journal papers in these research areas. $\mathrm{He}$ is a chartered engineer and a fellow of the IET. He was the general chair of the IEEE International Conference on Solid Dielectrics 2007 that was held in Winchester, Hampshire, UK. He is also secretary of the IEEE Dielectrics and Electrical Insulation Society, as well as an associate editor of the IEEE Transactions on Dielectrics and Electrical Insulation.

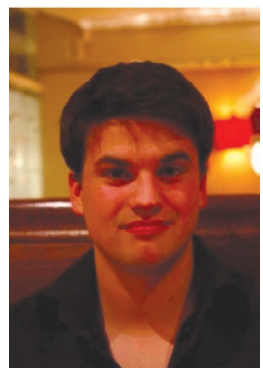

Ben Strawbridge is currently studying at the University of Southampton, School of Electronics and Computer Science, for a master's degree in electromechanical engineering. He is a student member of the IET. His interests include robotics, automotive engineering, and partial discharge.

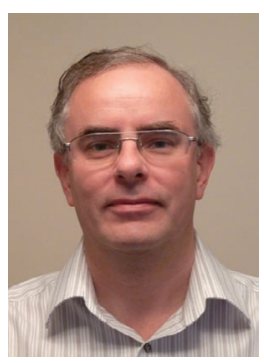

Paul Jarman was born in London on September 27, 1962, and graduated from Cambridge University in 1984 with an Honours Degree in electrical science. He joined the Central Electricity Generating Board, Research Division, working on, amongst other projects, FRA testing of transformers. In 1990, he joined the National Grid as a transformer engineer, becoming head of transformers in 1998. Since 2001, Jarman has been National Grid's technical specialist for transformers now within the Asset Management group. Jarman is chairman of IEC TC14, the international committee for power transformer standards; is the UK regular member of CIGRE study committee A2 for transformers; and has recently been the convenor of a CIGRE group on transformer monitoring. He is a chartered electrical engineer and member of the IET. 